\title{
Size functions
}

\author{
by \\ Niel Shell (New York)
}

\begin{abstract}
We introduce the notion of a nonarchimedean size function similar to the notion of a size function introduced by Marcos. We describe a class of ring topologies on fields that are complete, neither first countable nor locally bounded, but have topologically nilpotent elements.
\end{abstract}

Two types of valuations are commonly considered: real-valued valuations (also called absolute values) and nonarchimedean valuations (also called Krull valuations). In [3] Marcos introduced axioms for a function which we will call a real-valued size function. We introduce the notion of a nonarchimedean size function. As for valuations, the classes of real and nonarchimedean size functions overlap, but neither subsumes the other.

Marcos introduced size functions to construct topologies which yield an affirmative answer to a thirty year old open question in [2]: Do there exist topological fields which are not locally bounded but have topologically nilpotent elements? We describe here another class of ring topologies on fields with these properties.

A nonzero element $x$ in a topological ring is called topologically nilpotent if $x^{n} \rightarrow 0$. $A^{*}$ denotes the set of nonzero elements of a subset $A$ of an additive group, and $G_{\geq a}$ (resp. $G_{>a}$ ) denotes the set of elements greater than or equal to (resp. strictly greater than) $a$ in an ordered group $G$. In an ordered group (in particular, the real numbers) $a \vee b$ (resp. $a \wedge b$ ) denotes the larger (resp. smaller) of $a$ and $b$.

Definition 1. A real-valued size function on a $\operatorname{ring} A$ is a function $N$ : $A \rightarrow[1, \infty)$ such that

2000 Mathematics Subject Classification: Primary 54H13.

Key words and phrases: size function, topologically nilpotent element, locally bounded topology.

Research for this work was supported by the Research Foundation of the City University of New York via Grant Number 63458-00 32. 
(N1) $N(0)=1$;

(N2) $N(-a)=N(a)$;

(N3) $N(a+b) \leq N(a)+N(b)$;

(N4) $N(a b) \leq N(a)+N(b)$;

(N5) for each $g \geq 1$ there exists $a \in A$ such that $N(a) \geq g$.

A real-valued size function is called nonarchimedean if

$\left(\mathrm{N} 3^{\prime}\right) N(a+b) \leq N(a) \vee N(b) ;$

$\left(\mathrm{N} 4^{\prime}\right) N(a b) \leq N(a) \vee N(b)$.

Condition (N1) is dispensable, in the sense that the topologies induced in [3] by functions satisfying (N1)-(N5) can be induced by functions satisfying only (N2)-(N5). Indeed, in [3] Marcos requires $N(0)=2$, except for size functions that satisfy conditions $\left(\mathrm{N} 3^{\prime}\right)$ and $\left(\mathrm{N} 4^{\prime}\right)$.

The topology constructed in Section 3 of [3] is described in Theorem 1 below. We have replaced statements in [3] using logarithms with corresponding ones using exponents in order to make the analogy with Theorem 2 more apparent.

THEOREM 1. Let $N$ be a real-valued size function on a field $F$. Let $\gamma$ : $(1, \infty) \rightarrow \mathbb{R}$ be a strictly decreasing function such that $\lim _{t \rightarrow 1+} \gamma(t)=\infty$. Let $R$ consist of all formal series $\sum a_{n} X^{n} \in F[[X]]$ such that, for all $t>1$, there exists $m$ such that $N\left(a_{n}\right) \leq t^{n}$ for $n \geq m$. Then $R$ is a local ring whose maximal ideal consists of all series in $R$ with constant coefficient equal to 0 , and $K=\bigcup_{d \geq 0} X^{-d} R$ is the quotient field $R\left(R^{*}\right)^{-1}$. The sets

$$
V_{t}=\left\{\sum a_{n} X^{n} \in R: a_{n}=0 \text { for } n<\gamma(t) \text { and } N\left(a_{n}\right) \leq t^{n} \text { for } n \geq \gamma(t)\right\} \text {, }
$$

$t>1$, form a neighborhood base at zero for a complete field topology on $K$. This topology is not locally bounded, and $X$ is topologically nilpotent in this topology. The topology is independent of the particular choice of $\gamma$.

DeFinition 2. Let $G$ be an additive totally ordered group. An arbitrary element of $G_{>0}$ is labeled as 1 . Since no multiplication is defined, the label 1 is not intended to have any significance except to match notation in the real-valued case. A nonarchimedean $G$-valued size function on a $\operatorname{ring} A$ is a function $N: A \rightarrow G_{\geq 1}$ which satisifies (N1), (N2), (N3'), (N4') and (N5).

If $N$ is a nonarchimedean $G$-valued size function and $g \in G_{\geq 1}$, then $A_{g}=$ $\{a \in A: N(a) \leq g\}$ is a proper subring of $A$. The rings $A_{g}$ increase with $g$ and have union $A$. Conversely, given an increasing indexed set $\left\{A_{g}\right\}_{g \in G>0}$ of proper rings whose union is $A$ and given a cofinal well ordered subset $P$ of $G_{>0}$ whose smallest element we label as 1 , then letting $N(a)$ be the least element of $\left\{p \in P: a \in A_{p}\right\}$ defines a nonarchimedean size function. 
A nonarchimedean size function $N$ on a field satisfies the condition

(N6) $N(1)=1$ and $N\left(a^{-1}\right)=N(a)$ for all $a \neq 0$

if and only if $\{a \in A: N(a) \leq g\}$ is a field for each $g \in G_{\geq 1}$.

ExAMPLE. Let $F_{0}$ be a field and let $P$ be a well-ordered cofinal subset of positive elements of an ordered group $G$. Let 1 denote the least element of $P$, and let $T=\left\{t_{p}: p \in P \backslash\{1\}\right\}$ be a set of elements algebraically independent over $F_{0}$. Let $F=F_{0}(T), T_{p}=\left\{t_{q}: q \leq p\right\}$ (so $T_{1}=\emptyset$ ), and define $N(a)$ to be the least element of $\left\{p \in P: a \in F_{0}\left(T_{p}\right)\right\}$. Then $N$ is a nonarchimedean $G$-valued size function on $F$ satisfying (N6).

A nonarchimedean $G$-valued size function on a field $F$ may be used to induce a ring topology on a subfield of the field $F((G))$ of formal power series in somewhat the same way as for real-valued size functions. (See [1] or [5, Appendix B] for basic information about power series fields.)

The following result follows immediately from [8] (see also [5, Th. 2.3.2], [6] and [7]):

LEMma 2.1. Let $R$ be a ring with identity and with quotient field $K$, and let $\mathcal{T}$ be a nondiscrete ring topology on $R$. If the filter $\mathcal{B}(\mathcal{T})$ of $\mathcal{T}$ neighborhoods of zero satisfies the condition $x U \in \mathcal{B}(\mathcal{T})$ for all $x \in R^{*}$ and $U \in \mathcal{B}(\mathcal{T})$, then $\mathcal{B}(\mathcal{T})$ is a neighborhood base at zero for a nondiscrete ring topology on $K$.

THEOREM 2. Let $F$ be a field with a nonarchimedean $G$-valued size function $N$ such that $N(1)=1$ and $N\left(a^{-1}\right)=N(a)$ for $a \neq 0$. The set

$$
R=\left\{\sum a_{g} X^{g} \in F[[G]]: N\left(a_{g}\right) \leq g \vee 1 \text { for all } g \in G_{\geq 0}\right\}
$$

is a local ring whose maximal ideal consists of all series in $R$ with constant coefficient equal to 0 ; and $K=\bigcup_{d \geq 0} X^{-d} R$ is the quotient field $R\left(R^{*}\right)^{-1}$.

The sets $U_{h}$, where, for $h \geq 1$,

$$
\begin{aligned}
& U_{h}=\left\{\sum a_{g} X^{g} \in F[[G]]: a_{g}=0 \text { for all } g<h,\right. \\
& \text { and } \left.N\left(a_{g}\right) \leq g-h+1 \text { for all } g \geq h\right\},
\end{aligned}
$$

are ideals in $R$, and $\left\{U_{h}\right\}_{h \geq 1}$ is a neighborhood base at zero for a nondiscrete complete locally bounded Hausdorff field topology on $K$.

Proof. We will verify the following:

(1) $R$ is an additive group;

(2) $R R \subset R$;

(3) the inverse of an element in $R$ which has nonzero constant coefficient is again in $R$;

(4) $R\left(R^{*}\right)^{-1}=K$; 
(5) $U_{h}$ is an additive group for $h \geq 1$;

(6) $R U_{h} \subset U_{h}$ for $h \geq 1$;

(7) $X^{-d} U_{h+d} \subset U_{h}$ for $d \geq 0, h \geq 1$;

(8) $\left(1+U_{h}\right)^{-1}=1+U_{h}$ for $h \geq 1$.

From (1)-(3) it will follow that $R$ is a local ring whose maximal ideal consists of the series with constant coefficient equal to zero; (5) and (6) state that the sets $U_{h}$ are (clearly nonzero) ideals of $R$. Obviously $\left\{U_{h}\right\}$ is decreasing and $\bigcap\left\{U_{h}: h \geq 1\right\}=\{0\}$. Thus, the sets $U_{h}$ form a neighborhood base at zero for a nondiscrete Hausdorff ring topology on $R$. Using (4), (6), (7) and Lemma 2.1, we see that the sets $U_{h}$ also are a neighborhood base at zero for a ring topology on $K$ : Given $x \in R^{*}$, write $x^{-1}=X^{-d} y$, where $d \geq 0$ and $y \in R$. Then $X^{-d} y U_{h+d} \subset U_{h}$ or, equivalently, $U_{h+d} \subset x U_{h}$. By (8), inversion is continuous. The topology is locally bounded, since, by the definition of $U_{h}, X^{h-1} U_{1} \subset U_{h}$ for $h \geq 1$ (see [5, Th. 4.1.3]). Completeness will be verified after (1)-(8) are proved.

$(1),(5)$ and $(7)$ are obvious. The remaining statements all require consideration of the coefficient $c_{g}$ of $X^{g}$ in the product $x y$ of the series

$$
x=\sum a_{g} X^{g}, \quad y=\sum b_{g} X^{g},
$$

where $a_{g}=b_{g}=0$ for $g<0$. For some positive integer $n$, which depends on $g, c_{g}=a_{g_{1}} b_{g_{1}^{\prime}}+a_{g_{2}} b_{g_{2}^{\prime}}+\cdots+a_{g_{n}} b_{g_{n}^{\prime}}$, where, if $c_{g} \neq 0$, then $a_{g_{i}} b_{g_{i}^{\prime}} \neq 0$ for any $i$, and $g_{i}, g_{i}^{\prime} \geq 0$ and $g_{i}+g_{i}^{\prime}=g$; thus $g_{i}, g_{i}^{\prime} \leq g$.

(2) If $x, y \in R$, then $N\left(a_{g_{i}}\right) \leq g_{i} \vee 1 \leq g \vee 1$ and $N\left(b_{g_{i}^{\prime}}\right) \leq g_{i}^{\prime} \vee 1 \leq g \vee 1$, so $N\left(c_{g}\right) \leq g \vee 1$.

(3) For $x \in R$ with constant coefficient not zero, $y=x^{-1} \in R[[G]]$. We verify by induction on the index $g$ that $N\left(b_{g}\right) \leq g \vee 1$. We label indices so that $g_{1}^{\prime}<g_{2}^{\prime}<\cdots<g_{n}^{\prime}$. If $g=0$, then $n=1, g_{1}=g_{1}^{\prime}=0, b_{0}=a_{0}^{-1}$ (since $x y=1)$ and $N\left(b_{0}\right)=N\left(a_{0}^{-1}\right)=N\left(a_{0}\right) \leq 1$. We assume that $N\left(b_{k}\right) \leq k \vee 1$ for $k<g$. For $g>0, c_{g}=0$ (since $\left.x y=1\right)$. If $b_{g}=0$ we are done. Otherwise, we may take $g_{n}^{\prime}=g$ and $g_{n}=0$, and

$$
N\left(b_{g}\right)=N\left(-a_{0}^{-1}\left[a_{g_{1}} b_{g_{1}^{\prime}}+\cdots+a_{g_{n-1}} b_{g_{n-1}^{\prime}}\right]\right),
$$

and $N\left(b_{g}\right) \leq g \vee 1$ by the inductive hypothesis.

(4) Certainly $K \subset R\left(R^{*}\right)^{-1}$ and $K$ is a ring. Upon verifying that $z^{-1} \in K^{*}$ for $z \in K^{*}$, we find that $K$ is a field containing $R$ and, hence, containing the smallest field $R\left(R^{*}\right)^{-1}$ containing $R$. With $d, h \geq 0, N\left(a_{g}\right) \leq g \vee 1$ and $a_{h} \neq 0$, we may write

$$
z=X^{-d} \sum_{g \geq h} a_{g} X^{g}=X^{-d+h} \sum_{g \geq h} a_{g} X^{g-h}=X^{-d+h} \sum_{g \geq 0} a_{g+h} X^{g} .
$$

If we write $z^{-1}=X^{d-h} \sum_{g \geq 0} b_{g} X^{g}$, where $b_{g} \in F$, it follows, as in (3), from 
$N\left(a_{g+h}\right) \leq(g+h) \vee 1$ for all $g$ that $N\left(b_{g}\right) \leq(g+h) \vee 1$ for all $g$. Thus,

$$
z^{-1}=X^{d-h} X^{-h} \sum_{g \geq h} b_{g} X^{g+h} \in X^{d-2 h} R .
$$

(6) If $x \in R, y \in U_{h}$ and $c_{g} \neq 0$, then $g \geq h$ and $g_{i}^{\prime} \geq h$ for all $i$. Then $N\left(a_{g_{i}}\right) \leq g_{i} \vee 1=\left(g-g_{i}^{\prime}\right) \vee 1 \leq(g-h) \vee 1$. It follows that $N\left(c_{g}\right) \leq g-h+1$.

(8) Suppose $x \in 1+U_{h}$, where $h \geq 1$, and $y=x^{-1}$. Then $b_{0}=1$ and $b_{g}=0$ for $0<g<h$. For $g \geq h, c_{g}=0$ (since $x y=1$ ) and, as in the proof of $(3), N\left(b_{g}\right) \leq g-h+1$. Thus, $\left(1+U_{h}\right)^{-1} \subset 1+U_{h}$. Taking the inverse of both sides reverses the containment.

Finally, suppose that $x_{\lambda}=\sum a_{g}^{\lambda} X^{g}, \lambda \in \Lambda$, is a Cauchy net in $R$. Now, $x_{\lambda}-x_{\mu} \in U_{h}$ implies $a_{g}^{\lambda}=a_{g}^{\mu}$ for $g<h$. Thus, $a_{g}^{\lambda}$ is eventually constant for any fixed $g$; call the eventual value $a_{g}$. One readily verifies $x=\sum a_{g} X^{g} \in R$ and $x_{\lambda} \rightarrow x$. If $\left\{x_{\lambda}\right\}_{\lambda \in \Lambda}$ is a Cauchy net in $K$ then, for some $\mu \in \Lambda$, $\left\{x_{\lambda}-x_{\mu}\right\}_{\lambda \geq \mu}$ is a Cauchy net in $R$.

\section{References}

[1] L. Fuchs, Partially Ordered Algebraic Systems, Pergamon Press, Oxford, 1963.

[2] J. Heine, Existence of locally unbounded topological fields, and field topologies which are not the intersection of bounded ring topologies, J. London Math. Soc. 5 (1972), 481-487.

[3] J. E. Marcos, Locally unbounded topological fields with topological nilpotents, Fund. Math. 173 (2002), 21-32.

[4] - Erratum to "Locally unbounded topological fields with topological nilpotents", ibid. 176 (2003), 95-96.

[5] N. Shell, Topological Fields and Near Valuations, Monographs Textbooks Pure Appl. Math. 135, Dekker, New York, 1990.

[6] - Residue class topologies, in: Papers on General Topology and Applications (Flushing, NY, 1992), Ann. New York Acad. Sci. 728, New York Acad. Sci., New York, 1994, 339-345.

[7] - Direct topologies from discrete rings, III, J. Pure Appl. Algebra 173 (2002), 213-233.

[8] H. Weber, Unabhängige Topologien, Zerlegung von Ringtopologien, Math. Z. 180 (1982), 379-393.

The City College of New York (CUNY)

Convent Avenue at 138th Street

New York, NY 10031, U.S.A.

E-mail:nsxcc@cunyvm.cuny.edu

Received 18 November 2002;

in revised form 21 June 2004 